\title{
Synthesis of 5-Aryl-3-(5-Bromo-3-Benzofuran-2-yl)-1-Pyrazole as Antimicrobial Agent.
}

\author{
Vrushali Patil ${ }^{1}$, Ashish Asrondkar ${ }^{1}$, Shantanu Pande ${ }^{1}$, A.S.Bobade ${ }^{1}$, \\ A.S.Chowdhary ${ }^{1}$ \\ ${ }^{I}$ Department of Chemotherapy, Haffkine Institute for Training, Research and Testing, India
}

\begin{abstract}
In this work, we evaluate the biological activities of some new derivatives of benzofuran which can be used as effective anti-microbial agents. The recent reviews of literature have highlighted the attention of medicinal chemists because of their diverse biological activities and profound efficacy. Clinically potent benzofurans generated interest to construct a system which possesses anti-bacterial and anti-fungal activity. Furan and benzofuran are associated with wide spectrum of biological activity. In the view of these, an effort was made to check some synthesized compounds for their anti-microbial activity.

In present study, pyrazoline derivatives were synthesized; 5-bromo-3-methyl acetophenone undergoes ring formation in presence of chloroacetone to form benzofuran which further forms chalcone on treatment with substituted benzaldehyde. This intermediate on treatment with hydrazine hydrate results into pyrazoline. Further it reacts with various benzoyl chlorides to form the titled product. Synthesized compounds have been confirmed on the basis of spectral studies and analytical data. All the compounds were screened for their invitro anti-bacterial activity against Gram positive Staphylococcus aureus ATCC3750 and Gram negative Salmonella typhi NCTC786, anti-fungal strains of Candida albicans ATCC10233 and Aspergillus niger ATCC 16404 using tube dilution method showing moderate activity.
\end{abstract}

Keywords : Anti-microbial activity, Benzofuran, Pyrazole.

\section{INTRODUCTION}

Benzofuran ${ }^{1}$ compounds are ubiquitous in nature, particularly among plant kingdom. Often such natural products possessing benzofuran nucleus are endowed with useful pharmacological properties. This has generated enormous interest in synthetic products containing benzofuran nucleus and has resulted in the development of benzofuran chemistry during the last several years. Benzofurans are bicyclic ring system with multiple applications. The Literature indicates that compounds having Benzofuran ${ }^{2}$ nucleus possess broad range of biological activities, like Griseofulvin as antifungal; Amiodarone as antiarrythmic; Benzbromarone as uricosuric; Cloridarol as vasodilator; Oxetorone as antimigraine agent.

Pyrazolines are nitrogen-containing heterocyclic compounds, well known for their pronounced biological activity. These biological activities include antibacterial ${ }^{3}$, antifungal ${ }^{4}$, herbicidal ${ }^{5}$ and insecticidal activities ${ }^{6}$. It was demonstrated that the combination of pyrazole with azole ring, linked to each by one sigma bond, led to more biologically active targets; for example, pyrazolylthiazoles showed excellent antimicrobial activities $^{7}$.

Pyrazole $^{8}$ derivatives have a long history of application in Agrochemicals and Pharmaceutical industry as herbicides and active pharmaceuticals. The wide variety of Nitrogen containing heterocyclic compounds that have been exploited to develop pharmaceutically important molecules such as Pyrazole and their derivatives are important due to their diverse biological activities such as anti-fungal ${ }^{9}$, anti-bacterial ${ }^{10}$ and others.

The prevalence of pyrazole cores in biologically active molecules has stimulated the need for elegant and efficient ways to make these heterocyclic lead. These findings encourage us to undertake the synthesis of pyrazolene-benzofuran ring system, in the hope that they could have some promising biological interest.

\section{MATERIALS AND METHODS}

The synthesized compounds were screened for their anti-bacterial and anti-fungal activities. The various screenings carried out include the in vitro study against Gram positive Staphylococcus aureus ATCC 3750, Gram negative Salmonella typhi NCTC 786 and fungal strain Candida albicans and Aspergillus niger. The Minimum Inhibitory Concentration (MIC) was determined using tube dilution method according to the standard procedure $^{11}$. DMSO was used as a solvent with appropriate controls. Ampicillin (MIC $=0.01 \mu \mathrm{g} / \mathrm{ml}$ against Gram positive $\boldsymbol{S}$. aureus), Trimethoprim (MIC $=1 \mu \mathrm{g} / \mathrm{ml}$ against Gram negative $\boldsymbol{S}$. typhi) were used as standard drugs for anti-bacterial screening and Miconazole was used as anti-fungal standard drug (MIC $=0.01$ $\mu \mathrm{g} / \mathrm{ml})$. 


\subsection{EXPERIMENTAL}

\subsubsection{Synthesis of 5- Bromo-3-methyl-2acetyl benzofuran (I)}

To stirred mixture of $2.15 \mathrm{~g}(0.01 \mathrm{~mole})$ of 5-Bromo-2-hydroxy acetophenone and $3.45 \mathrm{~g}(0.025 \mathrm{~mole})$ of anhydrous $\mathrm{K}_{2} \mathrm{CO}_{3}$ in dry acetone for 30 mins. $1.0 \mathrm{ml}(0.01 \mathrm{~mole})$ of chloroacetone was added drop wise over a period of 10 mins. Reaction mixture was stirred for 3hours. Allow cooling and pour into crushed ice. Solid thus separated was filtered washed with $20 \mathrm{ml}$ cold water, crystallized with ethanol.

Yield: $60 \%$, M.P: $183^{\circ} \mathrm{C}$, Color \& Nature: white crystalline solid. ,CHN Found C\%: $52.45 \mathrm{H} \%: 3.73$. Calculated C\%:52.20 H\%:3.58; IR( $\mathrm{KBr}) \mathrm{cm}^{-1}: 1724$ (CO stretching), 585 (CBr stretching), $2892\left(\mathrm{CH}_{3}\right.$ aliphatic stretching), 2078 (Aromatic C-H stretching), 1640 (alkene $\mathrm{C}=\mathrm{C}$ stretching) $;{ }^{1} \mathrm{H}$ NMR (DMSO) 2ppm : 7.12-7.84 $(3 \mathrm{H} \mathrm{Ar}-\mathrm{H}, \mathrm{m}), 2.0(3 \mathrm{H}, \mathrm{s}), 3.2(3 \mathrm{H}, \mathrm{s})$

\subsubsection{Synthesis of 5-bromo-2-cinnamoyl-3-methyl Benzofuran (II)}

5-Bromo-2-acetylbenzofuran (1) $2.52 \mathrm{~g}(0.01 \mathrm{~mole})$ was stirred in $10 \mathrm{ml}$ of anhydrous ethyl alcohol. $1.40 \mathrm{~g}(0.01 \mathrm{~mole})$ of 3 -chlorobenzaldehyde was added to the reaction mixture at room temperature. This reaction mixture was cooled for 3 hours and contents allowed standing overnight. The solution was diluted with water and neutralized with dilute $\mathrm{HCl}$. The off-white colored solid thus separated was collected, dried and crystallized from ethanol.

Yield: $55 \%$, M.P: $135^{\circ} \mathrm{C}$, Color \& Nature: off white crystalline solid;CHN Found C\%: $57.68 \mathrm{H} \%$ : 2.94. Calculated $\mathrm{C} \%$ : $57.55 \mathrm{H} \%$ : 3.22; IR ( $\mathrm{KBr}) \mathrm{cm}^{-1}$; 585 (C-Br stretching), 594 (C-Cl stretching), 1640 (alkene $\mathrm{C}=\mathrm{C}$ stretching), $1122\left(\mathrm{C}=\mathrm{O}\right.$ stretching), 2010 (Ar stretching); ${ }^{1} \mathrm{H}$ NMR (DMSO) 2ppm : 7.12-7.84 (3H, Ar-H, m), $2.7(1 \mathrm{H}, \mathrm{d}), 2.9(1 \mathrm{H}, \mathrm{d})$

\subsubsection{Synthesis of 5-(3-Chlorophenyl)-3-(5-bromo-3-methylbenzofuran-2-yl-1H-pyrazolines (III)}

A mixture of 3.91g (0.01 mole) of 5-Chloro-2-cinnamoyl-3-methyl benzofuran (11) and 2.5g (0.15 mole) of hydrazine hydrate in $50 \mathrm{ml}$ anhydrous ethanol was refluxed for 3 hours. Excess of solvent was distilled off. Pour on to crush ice, solids collected. Product crystallized from ethanol.

Yield: $75 \%$, M.P: $146^{\circ} \mathrm{C}$, Color \& Nature: off white crystalline solid;CHN Found C\%: $55.68 \mathrm{H} \%: 3.95$ N\%: 7.43. Calculated C\%:55.48 H\%: $3.62 \mathrm{~N} \%: 7.19$; IR (KBr) $\mathrm{cm}^{-1}$ : 585 ( C-Br stretching ), 594 (C-Cl stretching), 1175 ( $\mathrm{C}=\mathrm{O}$ stretching), 1690 ( $\mathrm{C}=\mathrm{N}$ stretching), 2010 (Ar stretching), 1100 (CN stretching), 1620 (NH Bending); ${ }^{1} \mathrm{H}$ NMR (DMSO) 2ppm : 6.17- 7.58 (7H, Ar-H stretching, $2.4(3 \mathrm{H}, \mathrm{s}) 5.2(1 \mathrm{H}, \mathrm{s}), 5.0(2 \mathrm{H}, \mathrm{s})$

\subsubsection{Synthesis of 5-(-3-Chlorophenyl)-3-(5-bromo-3-methyl benzofuran-2-yl)-1-(Substituted benzoyl)-pyrazoline. (IV)}

A mixture of $1.94 \mathrm{~g}(0.005$ mole) of $1 \mathrm{H}-$ pyraoline (111) and $0.6 \mathrm{ml}(0.005$ mole) of substituted benzoyl chloride in anhydrous pyridine $(10 \mathrm{ml})$ was refluxed for 1 hour. Allow cooling reaction mixture neutralized with cold dilute $\mathrm{HCl}$. The solid separated out; product was filtered off and crystallized from ethanol.

Yield: $55 \%$, M.P: $205^{\circ} \mathrm{C}$, Color \& Nature: off white amorphous solid.: CHN Found C\%: $56.67 \mathrm{H} \%: 3.36 \mathrm{~N} \%$ : 5.48. Calculated C\%: $56.92 \mathrm{H} \%$ : $3.22 \mathrm{~N} \%$ : 5.31; IR $(\mathrm{KBr}) \mathrm{cm}^{-1}$ : 3144 ( C-H stretching Ar), 1689 ( $\mathrm{C}=\mathrm{O}$ stretching), $1555(\mathrm{~N}=\mathrm{C}$ stretching pyrazoline), $1263(\mathrm{C}=\mathrm{O}=\mathrm{C}$ stretching benzofuran), 1065 ( $\mathrm{C}-\mathrm{F}$ stretching), 722 ( C-Cl stretching).; ${ }^{1} \mathrm{H}$ NMR (DMSO) 2ppm : $7.18-8.00$ ( 10H, Ar-H), $2.3(3 \mathrm{H}, \mathrm{s}), 5.1(2 \mathrm{H}, \mathrm{s})$.

\subsubsection{Synthesis of [ 3-(5- bromo-3-methyl-1-benzofuran-2-yl)-5-(4-chlorophenyl)-4,5-dihydro- $1 \mathrm{H}$ pyrazol-1-yl](4-chlorophenyl) methanone}

Yield:52\%, M.P: $218^{\circ} \mathrm{C}$, Color \& Nature: brown amorphous soild.: CHN Found C\%: 56.59 H\%: 3.06 N\%: 5.56. Calculated C\%: $56.92 \mathrm{H} \%: 3.22 \mathrm{~N} \%$ : 5.31; $\mathrm{IR}(\mathrm{KBr}) \mathrm{cm}^{-1}: 585$ (C-Br stretching), 2078 (Ar stretching), 1060 (-C-O- stretching), $2950\left(\mathrm{CH}_{3}\right.$ stretching), 1705 (C=O stretching), 560 (C-Cl).; ${ }^{1} \mathrm{H}$ NMR (DMSO) 2ppm: $7.18-8.00$ ( 10H, Ar-H), $2.3(3 \mathrm{H}, \mathrm{s}), 5.1(2 \mathrm{H}, \mathrm{s})$

\subsubsection{Synthesis of [ 3-(5- bromo-3-methyl-1-benzofuran-2-yl)-5-(4-chlorophenyl)-4,5-dihydro- $1 \mathrm{H}$ pyrazol-1-yl](3-fluorophenyl) methanone}

Yield: 67\%, M.P: $189^{\circ} \mathrm{C}$, Color \& Nature: brown amorphous solid : CHN Found C\%: 58.25 H\%: 3.54 N\%: 5.63 Calculated C\%: 58.65 H\%:3.32 N\%:5.47; IR (KBr) cm ${ }^{-1}$ : 585 (C-Br stretching), 2078 (Ar stretching), 1060 (-C-O- stretching), $2950\left(\mathrm{CH}_{3}\right.$ stretching), 1705 (C=O stretching), $560(\mathrm{C}-\mathrm{Cl}), 1300$ (C-F).; ${ }^{1} \mathrm{H}$ NMR (DMSO) 2ppm: $7.18-8.00$ ( 10H, Ar-H), $2.3(3 \mathrm{H}, \mathrm{s}), 5.1(2 \mathrm{H}, \mathrm{s})$ 
1.1.4.3 Synthesis of [ 3-(5- bromo-3-methyl-1-benzofuran-2-yl)-5-(4-chlorophenyl)-4,5-dihydro$1 \mathrm{H}$ pyrazol-1-yl](4-Hydroxyphenyl) methanone

Yield: $65 \%$, M.P: $193^{\circ} \mathrm{C}$, Color \& Nature: off white amorphous solid: CHN Found C\%:58.92 H\%: 3.37 N\%:5.61 Calculated C\%: 58.88 H\%: $3.53 \mathrm{~N} \%: 5.49$; IR (KBr) $\mathrm{cm}^{-1}: 585$ (C-Br stretching), 2078 (Ar stretching), 1060 (-C-O- stretching), $2950\left(\mathrm{CH}_{3}\right.$ stretching), $1705(\mathrm{C}=\mathrm{O}$ stretching), 560 (C-Cl), $2951(\mathrm{O}-\mathrm{H}$ stretching).; ${ }^{1} \mathrm{H}$ NMR (DMSO) 2ppm: $7.18-8.00$ ( 10H, Ar-H), $2.3(3 \mathrm{H}, \mathrm{s}), 5.1(2 \mathrm{H}, \mathrm{s}), 3.1(1 \mathrm{H}, \mathrm{s})$

1.1.4.4 Synthesis of [ 3-(5- bromo-3-methyl-1-benzofuran-2-yl)-5-(4-chlorophenyl)-4,5-dihydro$1 \mathrm{H}$ pyrazol-1-yl](2,4-dimethoxyphenyl) methanone

Yield: 47\%, M.P: $198^{\circ} \mathrm{C}$, , Color \& Nature: white amorphous solid: CHN Found C\%: 58.75 H\%: 3.99 N\%: 5.41 Calculated C\%: $58.64 \mathrm{H} \%$ : $3.80 \mathrm{~N} \%: 5.06$; $\mathrm{IR}(\mathrm{KBr}) \mathrm{cm}^{-1}: 585$ (C-Br stretching), 2078 ( $\mathrm{Ar}$

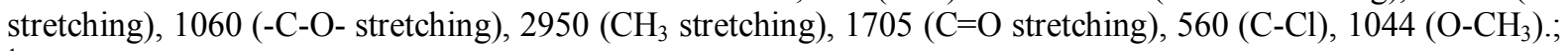
${ }^{1} \mathrm{H}$ NMR (DMSO) 2ppm: $7.18-8.00(10 \mathrm{H}, \mathrm{Ar}-\mathrm{H}), 2.3(3 \mathrm{H}, \mathrm{s}), 5.1(2 \mathrm{H}, \mathrm{s}), 5.2(6 \mathrm{H}, \mathrm{s})$.

1.1.4.5 Synthesis of [ 3-(5- bromo-3-methyl-1-benzofuran-2-yl)-5-(4-chlorophenyl)-4,5dihydro-1H pyrazol-1-yl](3-hydroxyphenyl)methanone

Yield: $63 \%$, M.P: $88^{\circ} \mathrm{C}$, Color \& Nature: White amorphous solid: CHN Found C\%: 59.65, H\%: 4.20, N\%: 5.41 Calculated C\%:58.70 H\%: 3.00 N\%: 4.90 ; IR (KBr): 3144 ( C-H stretching Ar), 1689 ( C=O stretching), $1555(\mathrm{~N}=\mathrm{C}$ stretching pyrazoline), $1263(\mathrm{C}=\mathrm{O}=\mathrm{C}$ stretching benzofuran), 722 ( $\mathrm{C}-\mathrm{Cl}$ stretching), 2950(OH stretching); ${ }^{1} \mathrm{H}$ NMR (DMSO) 2ppm : $7.18-8.00$ ( 10H, Ar-H), $2.3(3 \mathrm{H}, \mathrm{s}), 5.1(2 \mathrm{H}, \mathrm{s}), 2.7(1 \mathrm{H}, \mathrm{s})$

\subsubsection{Synthesis of [ 3-(5- bromo-3-methyl-1-benzofuran-2-yl)-5-(3-chlorophenyl)-4,5-dihydro- $1 \mathrm{H}$ pyrazol-1-yl](4-hydroxyphenyl)methanone}

Yield: $66 \%$, M.P: $128^{\circ} \mathrm{C}$, Color \& Nature: Off-white amorphous solid: CHN Found: C\%: $59.12 \mathrm{H} \%$ : 4.26 N\%: 5.50 Calculated: C\%: 58.30 H\%: 3.98 N\%: 5.00.;IR (KBr): 3144 ( C-H stretching Ar), 1689 ( C=O stretching), $1555(\mathrm{~N}=\mathrm{C}$ stretching pyrazoline), $1263(\mathrm{C}=\mathrm{O}=\mathrm{C}$ stretching benzofuran), 722 ( $\mathrm{C}-\mathrm{Cl}$ stretching), 2950(OH stretching); ${ }^{1} \mathrm{H}$ NMR (DMSO) 2ppm : $7.18-8.00$ ( 10H, Ar-H), $2.3(3 \mathrm{H}, \mathrm{s}), 5.1(2 \mathrm{H}, \mathrm{s}), 2.7(1 \mathrm{H}, \mathrm{s})$

\subsubsection{Synthesis of [ 3-(5- bromo-3-methyl-1-benzofuran-2-yl)-5-(3-chlorophenyl)-4,5-dihydro- 1H pyrazol-1-yl](4-hydroxyphenyl)methanone}

Yield: $51 \%$, M.P: $116^{\circ} \mathrm{C}$, Color \& Nature: white amorphous solid : CHN Found: C\%: 59.12 H\%: 4.02 N\%: 4.88 Calculated: C\%: $58.50 \mathrm{H} \%: 3.90$ N\%: 4.70: IR (KBr);3144 ( C-H stretching Ar), 1689 ( $\mathrm{C}=\mathrm{O}$ stretching), 1555 ( $\mathrm{N}=\mathrm{C}$ stretching pyrazoline), $1263\left(\mathrm{C}=\mathrm{O}=\mathrm{C}\right.$ stretching benzofuran), 722 ( $\mathrm{C}-\mathrm{Cl}$ stretching); ${ }^{1} \mathrm{H}$ NMR (DMSO) 2ppm: $7.18-8.00(10 \mathrm{H}, \mathrm{Ar}-\mathrm{H}), 2.3(3 \mathrm{H}, \mathrm{s}), 5.1(2 \mathrm{H}, \mathrm{s}), 2.7(1 \mathrm{H}, \mathrm{s})$ 
III. Table 1: Schematic Representation of Titled Compounds<smiles>CC(=O)c1cc(Br)ccc1O</smiles>
1-(5-bromo-2-hydroxyphenyl)ethanone

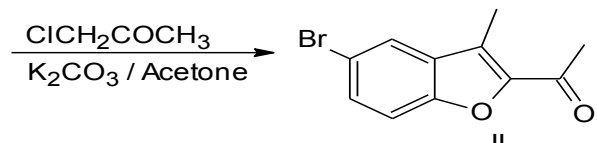
1-(5-bromo-3-methyl-1-benzofuran-2-yl)ethanone<smiles>[R]c1cccc(/C=C/C(=O)c2oc3ccc(Br)cc3c2C)c1</smiles>

(2E)-1-(5-bromo-3-methyl-1-benzofuran-2-yl)-3-phenylprop-2-en-1-one
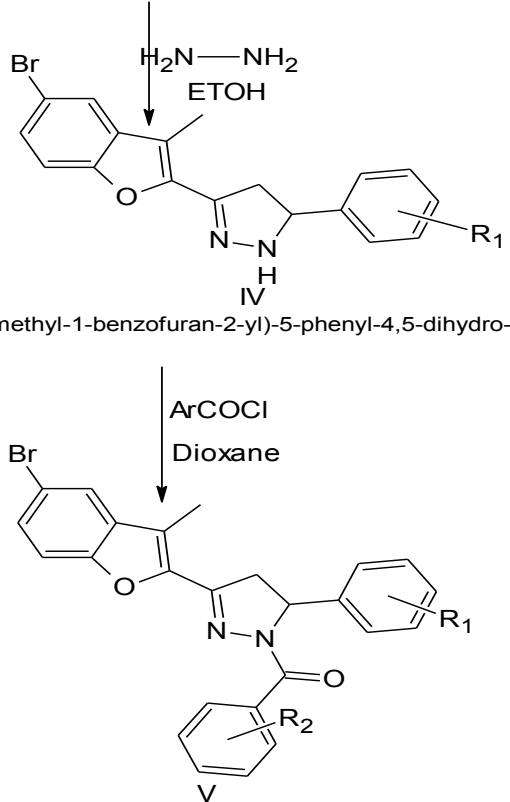

[3-(5-bromo-3-methyl-1-benzofuran-2-yl)-5-phenyl-4,5-dihydro-1H-pyrazol-1-yl](phenyl)methanone

\subsection{Table 2: Anti-microbial activity of synthesized compound}

\begin{tabular}{|l|l|l|l|l|l|l|}
\hline Sr.no & Substituent's & Antibacterial & Antifungal \\
\hline & $\mathrm{R}$ & $\mathrm{R}^{\prime}$ & S.aureus & S.typhi & C.albicans & A.niger \\
\hline 1 & 3-Chloro & 4-Chloro & 100 & 200 & 100 & 200 \\
\hline 2 & 4-Chloro & 4-Chloro & 200 & 200 & 50 & 200 \\
\hline 3 & 4-Chloro & 3-Fluoro & 100 & 100 & 100 & 100 \\
\hline 4 & 4-Chloro & 4-Hydroxy & 50 & 200 & 200 & 200 \\
\hline 5 & 4-Chloro & $\begin{array}{l}\text { 2,4- } \\
\text { Dimethoxy }\end{array}$ & 50 & 200 & 200 & N.A \\
\hline 6 & 4-Chloro & 3-Hydroxy & 100 & 100 & 100 & 100 \\
\hline 7 & 3-Chloro & 4-Hydroxy & 200 & N.A & 200 & N.A \\
\hline 8 & 3-Chloro & 3-Chloro & 100 & N.A & 200 & 100 \\
\hline
\end{tabular}

- Ampicillin (MIC- $0.04 \mu \mathrm{g} / \mathrm{ml}$ ) used as standard Staphylococcus aureus

- Trimethoprim (MIC- $0.01 \mu \mathrm{g} / \mathrm{ml}$ ) used as standard Salmonella typhi

- Miconazole (MIC- $6.25 \mu \mathrm{g} / \mathrm{ml}$ ) used as standard against Candida albicans and Aspergillus niger.

N.A.:- Not active at $200 \mu \mathrm{g} / \mathrm{ml}$. 


\section{RESULTS}

All the eight synthesized compounds (Table No.2) showed moderate activity upto $200 \mu \mathrm{g} / \mathrm{ml}$ among which 4-Hydroxy and 2, 4-Dimethoxy (Sr. no. 4\& 5) showed activity upto $50 \mu \mathrm{g} / \mathrm{ml}$ against Staphylococcus aureus. The 4-fluoro derivative (Sr. no. 3) exhibited activity at $100 \mu \mathrm{g} / \mathrm{ml}$ while the other remaining compounds exhibited activity upto $200 \mu \mathrm{g} / \mathrm{ml}$ against Salmonella typhi.

All the compounds showed moderate activity against fungal species of Candida albicans upto 100 $\mu \mathrm{g} / \mathrm{ml}$ exceptional of 2,4-Dimethoxy substitutions (Sr. no.5) with activity at $200 \mu \mathrm{g} / \mathrm{ml}$. The compounds with 4fluoro substitution (Sr. no 3) showed activity at $100 \mu \mathrm{g} / \mathrm{ml}$ while remaining others showed activity at $200 \mu \mathrm{g} / \mathrm{ml}$ against Aspergillus niger species except 2,4- Dimethoxy substitutions (compound no. 305) which is found inactive upto $200 \mu \mathrm{g} / \mathrm{ml}$.

\subsection{DISCUSSION}

The purpose of the present study was to examine whether molecular modifications might result in detection of new potential anti-microbial drugs. A series of compounds were prepared and assayed in variety of biological tests for anti-microbial activity. The data reported in table 2 shows that the effect of variation in chemical structure on activity was rather unpredictable. The substitution which appeared to be the most important for higher order of activity in the greatest number was test for p-chloroaryl group. The introduction of P-methoxy and P-hydroxy in aryl moiety of the pyrazole analogs produced compounds with anti-microbial properties.

\section{CONCLUSION}

In conclusion, the results of this investigation revealed that the observed substitution at Para position is better for enhancing anti-microbial activity. Obviously, the comparative evaluation of active compounds will require further studies; the data reported in this article may be a helpful guide for medicinal chemists.

\section{ACKNOWLEDGEMENTS}

The authors are thankful to SAIF, IIT, Powai, Mumbai for carrying out the CHN elemental analysis and also thankful to SAIF, Patiala University, Punjab for recording the NMR spectra.

\section{REFERENCES}

[1] S. Nizami, M. Gurumurthy, S. Chattarjee, and D Panda; Evaluation of Antimicrobial Potency of Some Synthesized Thiazolidin-4one substituted 1, 2, 4-triazoles, Journal of Advanced Pharmaceutical Research, 1, 2010, 26-35.

[2] M. Kamal , A. K. Shakya ,T.Jawaid ;Benzofuran: A New profile of biological activities, International Journal of Medical and Pharmaceutical Sciences, 1(03),2011,1-15

[3] H. Patel, V. Ugale, A. Ingale, and S. Bari; Synthesis and antimicrobial evaluation of pyrazo-thiazoles, Letters in Drug Design \& Discovery, 9, 2012, 840-847

[4] C.Y. Zhang, X.H. Liu, B.L. Wang, S.H. Wang, and Z.M. Li; Synthesis and antifungal activities of new pyrazole derivatives via 1,3-dipolar cycloaddition reaction, Chemical Biology and Drug Design, 75(5), 2010, 489-493.

[5] T. D. Sherman, M. V. Duke, R. D. Clark, E. F. Sanders, H. Matsumoto, and S. O. Duke,;Pyrazole phenyl ether herbicides inhibit protoporphyrinogen oxidase, Pesticide Biochemistry and Physiology, 40(3), 1991, 236-245.

[6] H. Song, Y. Liu, L. Xiong, Y. Li, N. Yang, and Q. Wang,;Design, synthesis, and insecticidal activity of novel pyrazole derivatives containing $\alpha$-hydroxymethyl-N-benzyl carboxamide, $\alpha$-chloromethyl-N-benzyl carboxamide, and 4,5-dihydrooxazole moieties, Journal of Agricultural and Food Chemistry, 60(6),2012,1470-1479.

[7] B. F. Abdel Wahab, A. Sediek, H. A. Mohamed, and G. E. A. Awad;; Novel 2-pyrazolin-1-ylthiazoles as potential antimicrobial agents, Letters in Drug Design \& Discovery, 10(2), 2013, 111-118.

[8] A.G Yadav, V.N Patil, A.S Bobade, S.V Athlekar, L.S Patil, A.S Chowdhary; Synthesis and Antimicrobial Activity of Some Benzimidazolyl Pyrazolone Derivatives, Asian Journal of Reasearch in Chemistry, 2(4), $2009,516$.

[9] V.H Babu, A Gupta, K.K. Srinivasan, K.S.R.Pai; Synthesis and Biological Evaluation of Benzofuran incorporated Benzodiazepines, Indian Journal of Heterocyclic Chemistry, 14, 2005, 267-268

[10] N.V Purohit, T. Sanghavi; Synthesis of Mesogenic Compounds with Dibenzofuran moiety as Antibacterial Agents, Indian Journal of Heterocyclic Chemistry, 14, 2005, 235-238.

[11] S.K Sahu, M. Banerjee, A. Samantray, C. Behera, M.A Azam; Synthesis, Analgesic, Anti-inflammatory and Antimicrobial Activities of Some Novel Pyrazoline Derivatives, Tropical Journal of Pharmaceutical Research,7(2), 2008, 961-968 